\title{
Incidence and Duration of Cumulative Bisphosphonate Use among Community-Dwelling Persons with or without Alzheimer's Disease
}

\author{
Miia Tiihonen $^{\mathrm{a}, \mathrm{b}, *}$, Heidi Taipale ${ }^{\mathrm{a}, \mathrm{c}}$, Antti Tanskanen ${ }^{\mathrm{d}, \mathrm{e}, \mathrm{f}}$, Jari Tiihonen ${ }^{\mathrm{d}, \mathrm{f}}$ and Sirpa Hartikainen ${ }^{\mathrm{a}, \mathrm{b}}$ \\ ${ }^{a}$ Kuopio Research Centre of Geriatric Care, School of Pharmacy, University of Eastern Finland, Kuopio, Finland \\ ${ }^{\mathrm{b}}$ School of Pharmacy, University of Eastern Finland, Kuopio, Finland \\ ${ }^{\mathrm{c}}$ Research Centre for Comparative Effectiveness and Patient Safety (RECEPS), University of Eastern Finland, \\ Kuopio, Finland \\ ${ }^{\mathrm{d}}$ Karolinska Institutet, Department of Clinical Neuroscience, Stockholm, Sweden \\ ${ }^{\mathrm{e}}$ National Institute for Health and Welfare, Helsinki, Finland \\ ${ }^{\mathrm{f}}$ University of Eastern Finland, Department of Forensic Psychiatry, Niuvanniemi Hospital, Kuopio, Finland
}

Handling Associate Editor: Diana Wucherer

Accepted 15 January 2016

\begin{abstract}
We studied the incidence and duration of cumulative bisphosphonate use among older Finnish women and men with or without Alzheimer's disease (AD). The MEDALZ-2005 cohort is a nationwide sample of all persons with clinically diagnosed AD on 31 December 2005 and their age-, gender-, and region of residence-matched control persons without AD. Information on bisphosphonate use by persons with an AD diagnosis and their controls without AD during 2002-2009 was obtained from the prescription register database containing reimbursed medications. A total of 6,041 (11.8\%) persons used bisphosphonates during the 8-year follow-up. Bisphosphonates were more commonly used among persons without AD $(n=3121,12.3 \%)$ than among persons with $\mathrm{AD}(n=2,920,11.2 \%)(p=0.001)$. The median duration of bisphosphonate use was 743 days (IQR). Among persons with AD, the median duration of use was 777 days (IQR) and among persons without $\mathrm{AD}, 701$ days (IQR) $(p=0.011)$. People without $\mathrm{AD}$ more often used bisphosphonate combination preparations including vitamin $\mathrm{D}$ than did people with $\mathrm{AD}(p<0.0001)$. Bisphosphonate use was more common among people without $\mathrm{AD}$ who had comorbidities, asthma/COPD, or rheumatoid arthritis compared with users with AD. Short-term users were more likely to be male, at least 80 years old, and not having AD. Although the incidence of bisphosphonate use was slightly higher among persons without $\mathrm{AD}$, the cumulative duration of bisphosphonate use was longer in persons with $\mathrm{AD}$. Short-term use was associated with male gender, older age, and not having AD.
\end{abstract}

Keywords: Alzheimer's disease, bisphosphonates, community dwelling, osteoporosis

\section{INTRODUCTION}

*Correspondence to: Miia Tiihonen, University of Eastern Finland, P.O.B 1627, FI-70211 Kuopio, Finland. Tel.: +358 40 5819051; Fax: +358 17162 131; E-mail: miia.tiihonen@uef.fi.
Bisphosphonates are considered one of the first-line medications for preventing osteoporotic fractures. Bisphosphonates have been considered 
both effective and cost-effective in preventing vertebral, peripheral, and hip fractures also in older populations [1-3]. Bisphosphonates are considered relatively safe [4]. However, oral bisphosphonates may cause gastrointestinal problems, esophagitis, and, in rare cases, atypical fractures of the femur. Thus, it is recommended that prescribers regularly review the need for treatment, especially after five or more years of use.

The risk of osteoporotic fracture is markedly higher among older people suffering from cognitive impairment and dementia, including Alzheimer's disease (AD). People with AD have a 2-3-fold risk of hip fracture compared with AD-free people [5]. Dementia and fracture risk are both increased through decreased mobility and impaired balance, smoking, alcohol use, and vitamin D deficiency [6]. Additionally, dementia is associated with an increased risk of falls and low bone mineral density (BMD) and hence, a greater risk of fracture. A lack of early recognition of osteoporosis risk through BMD tests and underutilization of osteoporosis medication have been associated with both older age and dementia [7-9].

As far as we know, no previous studies have investigated the incidence of bisphosphonate use among community-dwelling women and men with diagnosed AD. The objective of our study was to examine the incidence and duration of cumulative bisphosphonate use among Finnish community-dwellers with or without AD.

\section{MATERIALS AND METHODS}

The MEDALZ-2005 (Medication use and Alzheimer's disease) cohort encompasses all Finnish community-dwelling persons with a verified $A D$ diagnosis and alive on December 31, 2005 [10]. Persons with AD were identified from the Special Reimbursement Register maintained by the Social Insurance Institution (SII) in Finland, and one control matched by age, gender, and region of residence and without $\mathrm{AD}$ was identified for each person with diagnosis of AD. The cohort consists of 56,186 persons, of which 28,093 have AD. The cohort was followed up until the end of 2009. The diagnosis of AD was based on the NINCDS-ADRDA and DSM-IV criteria for AD [11]. The MEDALZ-2005 cohort includes persons with all stages of AD. The details of the MEDALZ-2005 cohort and registers are described by Tolppanen et al. [10]. No ethics committee approval was required as de-identified data were used.

Information on bisphosphonate use among persons with an $\mathrm{AD}$ diagnosis and their controls without $\mathrm{AD}$ in 2002-2009 was obtained from the prescription register. The Finnish prescription register includes information on all reimbursed prescription drug purchases classified according to the Anatomical Therapeutic Chemical classification system (ATC) and defined as daily dose (DDD), which is the assumed average maintenance dose per day for a drug used for its main indication in adults [12]. ATC codes M05BA (alendronate, etidronate, risedronate, ibandronate, zoledronate, pamidronate and clodronate) and M05BB (risedronate and calcium, alendronate and cholecalciferol) were included in the present study. Only bisphosphonate users who started the treatment in 2002-2009 (without treatment in 2001) and persons using bisphosphonates for fracture prevention were included. Thus, people with cancer diagnoses (breast cancer, prostate cancer, leukemia, and other malignant diseases of the blood and bone marrow, malignant diseases of the lymphatic system, cancer of the female genital organs, or other malignant tumors) $(n=2971)$ and possibly using bisphosphonates for treatment of cancer-related bone metastases or hypocalcaemia were excluded. After exclusion of persons with bisphosphonate use in 2001 or with cancer, the present study consisted of 51,292 persons, including 25,850 persons with $\mathrm{AD}$ and 25,422 controls. The follow-up ended on December 31, 2009, institutionalization, or death.

Short-term bisphosphonate users were defined as persons who had used bisphosphonates for less than 365 days but had an opportunity to use them for at least one year in terms of the follow-up time after starting drug use. Long-term use was defined as cumulative use for more than one year. One person may have had multiple bisphosphonate periods with either the same or different bisphosphonate substances. These were summed together for the calculation of cumulative duration of bisphosphonate use. In Finland, a maximum of 3 months of treatment per purchase may be dispensed at one time.

A mathematical modeling method was used to define the cumulative duration of bisphosphonate use [13]. Drug use periods (when continuous drug use started and ended) were defined from individual purchase histories using the purchased amount in DDDs, which is recorded for each purchase and for each drug (ATC code) in the prescription register. This method is based on calculation of temporal sliding averages 
from three subsequent purchases. Purchases were included in the same drug use period if the purchased amount of drug was enough to last to the next purchase when stockpiling, personal purchase regularity, and hospitalizations were taken into account. Use of each ATC code was modeled separately. When studying the cumulative duration of bisphosphonate use, overlapping periods of using bisphosphonate drug substances were combined for each person to retrieve "use of any bisphosphonate". Thus, during any period of bisphosphonate use persons were allowed to switch between drug substances and drug combinations if there were no breaks in the availability of the drug.

Charlson's comorbidity index [14] was calculated for each person at the start of the follow-up on January 1, 2006, based on the diagnoses recorded in the Special Reimbursement Register. The index included heart failure, coronary artery disease, diabetes type I or type II, chronic asthma or chronic obstructive pulmonary disease (COPD), disseminated connective tissue diseases, rheumatoid arthritis and other comparable conditions (score of 1); uremia requiring dialysis, severe anemia in connection with chronic renal failure, leukemia and other malignant diseases of blood and bone marrow including malignant diseases of the lymphatic system, and cancer including breast and prostate cancers, female genital tract cancer and malignant neoplasms (score of 2). The Charlson's indexes were classified as: 0,1 , and 2 or more.

Statistical analyses were performed with SAS Software 9.3 and SPSS 21.0. Descriptive statistical analyses were done for continuous variables using means, medians, and standard deviations (SD). A T-test was used to measure differences between means and a Kruskal-Wallis test was used to test differences between medians. Chi-squared tests for categorical variables were used to compare the characteristics of bisphosphonate users with and without AD. Univariate and multivariate logistic regression analyses (forward entry selection) was utilized to evaluate factors associated with short-term bisphosphonate use in comparison with long-term use.

\section{RESULTS}

The MEDALZ-2005 cohort included a total of $6041(11.8 \%)$ persons that had used bisphosphonates during the 8-year follow-up. Bisphosphonates were used by $15.8 \%(n=5519)$ of the women and $3.2 \%$ $(n=522)$ of the men. The mean age of the bispho- sphonate users was 80.8 years (54-99, SD 5.815). Mean age of male bisphosphonate users was 79.7 (SD 6.192) and mean age of female bisphosphonates users was 80.9 (SD 5.768) $(p<0.0001)$. Bisphosphonates were more commonly used among persons without $\mathrm{AD}(n=3121,12.3 \%)$ than among persons with $\mathrm{AD}$ $(n=2920,11.2 \%)(p=0.001)$. There were no significant gender differences in terms of AD diagnosis and bisphosphonate use. Of people with AD using bisphosphonates, 37\% $(n=1085)$ have started treatment before the diagnosis of AD. The prevalence of asthma/COPD and rheumatoid arthritis was higher among bisphosphonate users than among non-users.

The median duration of bisphosphonate use was 743 days (IQR). Among people with AD, the median duration of use was 777 days (IQR) and among people without $\mathrm{AD}, 701$ days (IQR) $(p=0.011)$. Characteristics of the bisphosphonate users with and without AD are presented in Table 1. Persons without AD more often used bisphosphonate combination preparations, including vitamin $\mathrm{D}$, than did persons with $\mathrm{AD}$ $(p<0.0001)$. Bisphosphonate use was more common among persons without $\mathrm{AD}$ who had comorbidities (Charlson's index 2 or more) and among those with asthma/COPD or rheumatoid arthritis compared with users with AD.

Of the bisphosphonate users, $32.5 \%(n=1966)$ had used bisphosphonates for less than one year. Of these persons, $89 \%(n=1746)$ were short-term users. In the multivariate model, factors associated with shortterm use were male gender, at least 80 years of age, and no AD diagnosis (Table 2). In the multivariate logistic regression analysis, cumulative use of five years or more, compared with those with shorter use, was associated with female gender (OR 9.66; 95\% CI, 6.93-13.45), rheumatoid arthritis (OR 2.98, CI 2.23-3.90), and having asthma/COPD (OR 1.85; 95\% CI, 1.45-2.36).

\section{DISCUSSION}

This is the first nationwide study focusing on the incidence and duration of cumulative bisphosphonate use among women and men with diagnosed AD. We found a slightly higher incidence of bisphosphonate use among persons without AD. Although there are no previous studies of incident use that focus on bisphosphonate use, the prevalence of any osteoporosis drug use has been negatively associated with cognitive decline among community-dwellers $[9,15]$. Previous studies have suggested that demen- 
Table 1

Characteristics of bisphosphonate users among persons with or without AD in the MEDALZ-2005 study

\begin{tabular}{|c|c|c|c|}
\hline & $\begin{array}{l}\text { All bisphosphonate } \\
\text { users with AD \% } \\
(n=2920)\end{array}$ & $\begin{array}{l}\text { All bisphosphonate } \\
\text { users without AD \% } \\
\qquad(n=3121)\end{array}$ & $\begin{array}{c}p \text {-value } \\
0.001\end{array}$ \\
\hline \multicolumn{4}{|l|}{ Age } \\
\hline Mean (SD) & $80.84(5.905)$ & $80.68(5.729)$ & 0.103 \\
\hline$<75$ & $13.3(389)$ & $13.4(417)$ & 0.964 \\
\hline 75-79 & $25.3(739)$ & $25.9(808)$ & 0.605 \\
\hline $80-84$ & $34.3(1003)$ & $35.6(1111)$ & 0.309 \\
\hline $85-89$ & $21.4(626)$ & $20.0(623)$ & 0.157 \\
\hline$\geq 90$ & $5.3(163)$ & $5.2(162)$ & 0.500 \\
\hline$\overline{\text { Female gender }}$ & $91.0(2656)$ & $91.7(2863)$ & 0.284 \\
\hline \multicolumn{4}{|l|}{ Bisphosphonate } \\
\hline M05BA & $88.2(2574)$ & $83.0(2591)$ & $<0.0001$ \\
\hline M05BB (Bisphosphonate+vitamin D) & $7.8(227)$ & $11.2(348)$ & \\
\hline Both M05BA and M05BB & $4.1(119)$ & $5.8(182)$ & \\
\hline Cumulative bisphosphonate use (with an opportunity to use) & $(76 \%, n=2226)$ & $(78 \%, n=2430)$ & \\
\hline$<$ one year & $32.1(715)$ & $42.4(1031)$ & $<0.0001$ \\
\hline $1-3$ years & $32.5(701)$ & $24.1(586)$ & \\
\hline$>3$ to 5 years & $19.3(430)$ & $16.3(397)$ & \\
\hline$>5$ years & $17.1(380)$ & $17.1(416)$ & \\
\hline \multicolumn{4}{|l|}{ Charlson's comorbidity index } \\
\hline 0 & $55.8(1629)$ & $51.4(1604)$ & $<0.0001$ \\
\hline 1 & $31.4(917)$ & $32.6(1019)$ & \\
\hline$\geq 2$ & $12.8(374)$ & $16.0(498)$ & \\
\hline \multicolumn{4}{|l|}{ Comorbidities } \\
\hline Asthma/COPD & $10.1(295)$ & $13.4(419)$ & $<0.0001$ \\
\hline Diabetes & $9.5(277)$ & $10.1(315)$ & 0.428 \\
\hline Rheumatoid arthritis & $7.9(231)$ & $10.0(321)$ & 0.005 \\
\hline Cardiac insufficiency & $10.4(305)$ & $11.6(363)$ & 0.142 \\
\hline Coronary heart disease & $22.9(651)$ & $22.3(7169)$ & 0.548 \\
\hline
\end{tabular}

$p$-value: Persons with AD using bisphosphonates compared with persons without AD using bisphosphonates; chi-square.

Table 2

Factors associated with short-term bisphosphonate use among persons with or without AD diagnoses in the MEDALZ-2005 study

\begin{tabular}{lcc}
\hline & $\begin{array}{c}\text { Univariate OR } \\
(95 \% \mathrm{CI})\end{array}$ & $\begin{array}{c}\text { Multivariate OR } \\
(95 \% \mathrm{CI})\end{array}$ \\
\hline AD diagnosis & $0.75(0.68-0.84)$ & $0.75(0.67-0.83)$ \\
Male gender & $1.73(1.44-2.08)$ & $1.77(1.48-2.13)$ \\
$\geq 80$ years of age & $1.36(1.29-1.44)$ & $1.16(1.04-1.29)$ \\
Charlson's & & \\
$\quad$ comorbidity index* & & \\
1 & $1.04(0.93-1.17)$ & \\
$\geq 2$ & $1.03(0.95-1.11)$ & \\
Asthma/COPD & $1.00(0.84-1.18)$ & \\
Rheumatoid arthritis & $0.88(0.73-1.07)$ & \\
\hline
\end{tabular}

Logistic regression model comparing short-term users with longterm users. *Charlson's comorbidity index in comparison with a score of 0 .

tia dominates other comorbidities and thus may lead to underdiagnosis $[9,16]$. Polypharmacy, concerns about potential or actual adverse effects, and limited life expectancy may also limit prescribing to persons with dementia.

The median cumulative duration of bisphosphonate use was longer in people with AD. People without $\mathrm{AD}$ discontinued bisphosphonate use more often within one year and more often used preparations including bisphosphonates in combination with vitamin $\mathrm{D}$ than did people with $\mathrm{AD}$, possibly because of better ability to communicate their needs and subjective adverse effects. Adherence problems and poor persistence of use are associated with bisphosphonate use [17]. One explanation for poor adherence and persistence may be the asymptotic nature of osteoporosis; bisphosphonate treatment aims to protect from future fractures, but has no immediate impact on symptoms or well-being. This problem has been observed among other asymptomatic chronic conditions such as hypertension or hypercholesterolemia [18]. In addition to direct experiences of adverse effects as well as costs, comorbidities, and the patient's beliefs appear to have an impact on whether osteoporosis drug use is continued. In the present study, persons with asthma/COPD or rheumatoid arthritis more often were bisphosphonate users and long-term users, presumably due to glucocorticoid use.

In the present study, the incidence of bisphosphonate use among men was rare (3\%), and short-term use was associated with male gender. Osteoporosis 
is associated in female gender and, thus, a majority of previous studies have mostly focused on female populations $[8,19]$. It has been suggested that male osteoporosis has been both unrecognized and undertreated, although mortality associated with fractures is even higher among men than women.

The major strength of our study is the nationwide sample of both older women and men who use bisphosphonates, with a long follow-up. Drug use was based on prescription register data, which have previously been reported to be reliable [20]. The present study describes the mathematically modeled duration of bisphosphonate treatment instead of a single-point cross-sectional design [13]. Further, we compared bisphosphonate use among persons with $\mathrm{AD}$ with their age-, gender-, and region of residence-matched comparison persons without AD. Persons with AD in our study had a clinically verified diagnosis of $\mathrm{AD}$.

In the present study, we did not know the indication for bisphosphonate use, e.g., whether it was used for primary or secondary prevention of osteoporosis. Another limitation was the fact that we did not capture confounders such as lifestyle factors (height, weight, smoking, and alcohol consumption), BMD and socioeconomic factors, or use of calcium/vitamin D supplements (available also over the counter in Finland). The severity of AD was not available in our register-based data. Unfortunately, we were not able to identify other dementia etiologies or mild cognitive impairment. Thus, some of the controls might have dementia due other causes than AD. However, $\mathrm{AD}$ covers at least $80 \%$ of the dementia cases [21].

In conclusion, although the incidence of bisphosphonate use was slightly higher among people without $\mathrm{AD}$, the cumulative duration of bisphosphonate use was longer in people with AD. Short-term use was associated with male gender, older age, and not having AD. More research on bisphosphonate use in relation to fracture history among people with or without $\mathrm{AD}$ is needed to identify possible differences in treatment practices.

\section{DISCLOSURE STATEMENT}

Authors' disclosures available online (http://jalz.com/manuscript-disclosures/15-1181r1).

\section{REFERENCES}

[1] Cranney A, Wells G, Willan A, Griffith L, Zytaruk N, Robinson V, Black D, Adachi J, Shea B, Tugwell P, Guyatt G (2002) Meta-analyses of therapies for postmenopausal osteoporosis. II. Meta-analysis of alendronate for the treatment of postmenopausal women. Endocr Rev 23, 508-516.

[2] Cranney A, Tugwell P, Adachi J, Weaver B, Zytaruk N, Papaioannou A, Robinson V, Shea B, Wells G, Guyatt G (2002) Meta-analyses of therapies for postmenopausal osteoporosis. III. Meta-analysis of risedronate for the treatment of postmenopausal osteoporosis. Endocr Rev 23, 517-523.

[3] Strom O, Borgstrom F, Sen SS, Boonen S, Haentjens P, Johnell O, Kanis JA (2007) Cost-effectiveness of alendronate in the treatment of postmenopausal women in 9 European countries - an economic evaluation based on the fracture intervention trial. Osteoporos Int 18, 1047-1061.

[4] Rizzoli R, Reginster J-Y, Boonen S, Breart G, Dietz-Perez A, Felsenberg D, Kaufman J-M, Kanis JA, Cooper C (2011) Adverse reactions and drug-drug interactions in the management of women with postmenopausal osteoporosis. Calsified Tissue Int 89, 91-104.

[5] Tolppanen A-M, Lavikainen P, Soininen H, Hartikainen S (2013) Incident hip fractures among community dwelling persons with Alzheimer's disease in a Finnish nationwide register-based cohort. PloS One 8, e59124.

[6] Friedman SM, Menzies IB, Bukata SV, Mendelson DM, Kates SL (2010) Dementia and hip fractures: Development of a pathogenic framework for understanding and studying risk. Geriatr Orthop Surg Rehabil 1, 52-62.

[7] McNally DN, Kenny AM, Smith JA (2007) Adherence of academic geriatric practitioners to osteoporosis screening guidelines. Osteoporos Int 18, 177-183.

[8] Johnell K, Fastbom K (2009) Undertreatment of osteoporosis in the oldest old? A nationwide study of over 700,000 older people. Arch Osteoporos 4, 17-23.

[9] Haasum Y, Fastborn J, Fratiglioni L, Johnell K (2012) Undertreatment of osteoporosis in persons with dementia? A population-based study. Osteoporos Int 23, 1061-1068.

[10] Tolppanen AM, Taipale H, Koponen M, Lavikainen P, Tanskanen A, Tiihonen J, Hartikainen S (2013) Use of existing data sources in clinical epidemiology: Finnish health care registers in Alzheimer's disease research - the Medication use among persons with Alzheimer's disease (MEDALZ-2005) study. Clin Epidemiol 5, 277-285.

[11] McKhann G, Drachman D, Folstein M, Katzman R, Price D, Stadlan EM (1984) Clinical diagnosis of Alzheimer's disease: Report of the NINCDS-ADRDA work group under the auspices of department of health and human services task force on Alzheimer's disease. Neurology 34, 939-944.

[12] WHO Collaborating Centre for Drug Statistics Methodology. The Anatomical Therapeutic Chemical Classification System. http://www.whocc.no/atc_ddd_index/. Accessed 13 Feb 2014

[13] Tanskanen A, Taipale H, Koponen M, Tolppanen AM, Hartikainen S, Ahonen R, Tiihonen (2015) From prescription drug purchases to drug use periods - a second generation method (PRE2DUP). BMC Med Inform Decis Mak 15, 21.

[14] Charlson ME, Pompei P, Ales KL, MacKenzie CR (1987) A new method of classifying prognostic comorbidity in longitudinal studies: Development and validation. J Chronic Dis 40, 373-383.

[15] Jachna CM, Shireman TI, Whittle J, Ellerbeck EF, Rigler SK (2005) Differing patterns of antiresorptive pharmacotherapy in nursing facility residents and community dwellers. $J \mathrm{Am}$ Geriatr Soc 53, 1275-1281.

[16] Brauner DJ, Muir JC, Sachs GA (2000) Treating nondementia illnesses in patients with dementia. JAMA 283, 3230-3235. 
[17] Imaz I, Zegarra P, Gonzalez-Enriquez J, Rubio B, Alcazar R, Amate JM (2010) Poor bisphosphonate adherence for treatment of osteoporosis increases fracture risk: Systematic review and meta-analysis. Osteoporos Int 21, 1943-1951.

[18] Vrijens B, Vincze G, Kristanto P, Urquhart J, Burnier M (2008) Adherence to prescribed antihypertensive drug treatments: Longitudinal study of electronically compiled dosing histories. BMJ 336, 1114.

[19] von Friesendorff M, McGuigan FE, Besjakov J, Åkesson $\mathrm{K}$ (2011) Hip fracture in men-survival and subsequent fractures: A cohort study with 22-year follow-up. $J$ Am Geriatr Soc 59, 806-813.

[20] Haukka J, Suvisaari J, Tuulio-Henriksson A, Lönnqvist J (2007) High concordance between self-reported medication and official prescription database information. Eur J Clin Pharmacol 63, 1069-1074.

[21] Alzheimer's Association (2014) Alzheimer's disease facts and figures. Alzheimers Dement 10, e47-92. 